\title{
EVIDENCE FOR PRIMORDIAL ABUNDANCE VARIATIONS IN THE GLOBULAR CLUSTER 47 TUC
}

Based on spectra of 106 cluster stars

\author{
B.F.W. CROKE \\ IESL-FORTH and the University of Crete \\ R.A. STATHAKIS AND R.D. CANNON \\ Anglo-Australian Observatory \\ J.E. HESSER \\ Dominion Astrophysical Observatory \\ AND \\ R.A. BELL \\ University of Maryland
}

The FOCAP multi-object system on the Anglo-Australian Telescope (AAT) has been used to observe 35 faint stars $\left(V>18, M_{v}>+4.6\right)$ and 83 stars with $\mathrm{V}<18$ in 47 Tuc. The large sample of stars well below the turnoff permits us to examine abundance variations in relatively unevolved stars for the first time. Our spectral resolution $(2.7 \AA)$ is greater than that used in previous studies.

Some evidence is found for significant scatter in the strength of the $3883 \AA \mathrm{CN}$ band (a $4.5 \sigma$ result) and the CH G band (2.5 $\sigma$ ) for stars with $+4.1<\mathrm{M}_{\mathrm{v}}<+5.1$. The $3883 \AA \mathrm{CN}$ and $\mathrm{CH}$ bands are anticorrelated for these stars.
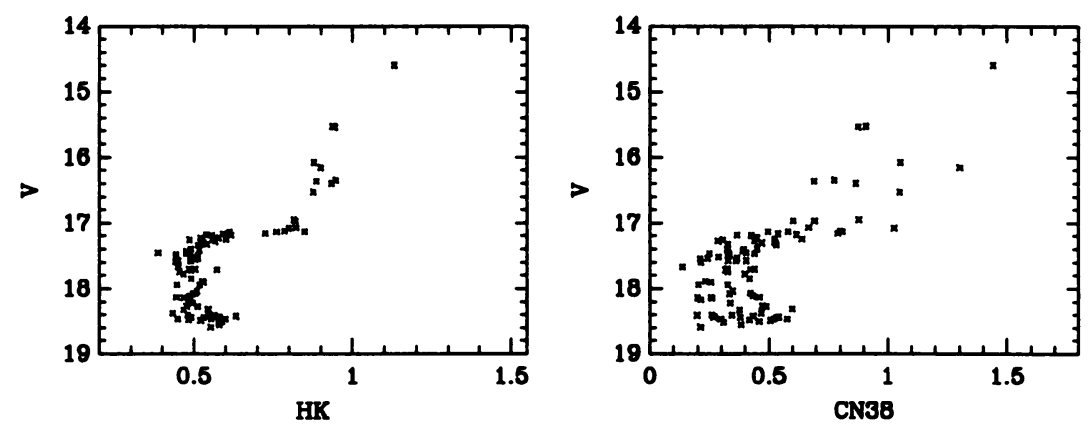

Figure 1. Observed Index-Magnitude Diagrams 